
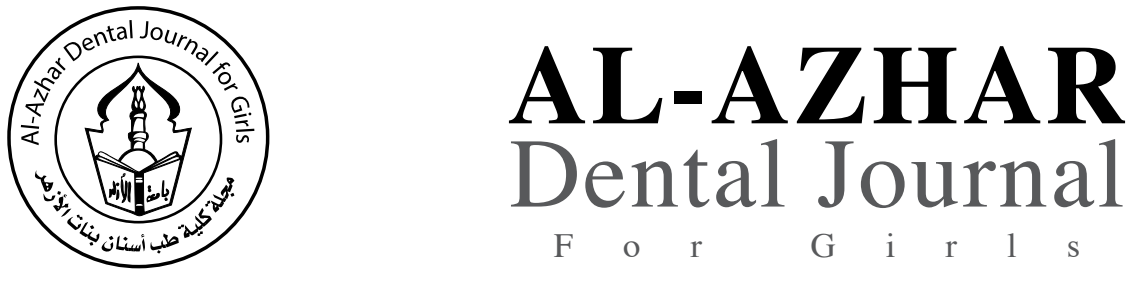

The Official Publication of The Faculty of Dental Medicine For Girls, Al-Azhar University Cairo, Egypt.

ADJ-for Grils, Vol. 4, No. 4, October (2017) - PP. 417:425

\title{
Assessment of the Smile Characteristics in Different Dentoalveolar Malocclusion Cases
}

\author{
Mohsena A. Abdarazik ${ }^{(1)}$, Samir A. Ibrahim ${ }^{(2)}$ and Tarek H. Marei $^{(3)}$
}

Codex : 48/1710

dentaljournal.forgirls@yahoo.com

\begin{abstract}
Objectives: This study was designed to assess the smile characteristics in different dentoalveolar malocclusion cases, by standardized photographic analysis. Subjects and methods: A sample of 132 subjects with age range from 18 to 24 years. The study groups were designed according to Angles Classification in to 4 groups. Standardized extraoral photographs at rest position, social smile, maximum smile and profile were taken for each subject. Smile analysis was done by identifying certain measurements digitally via software. Results: The upper and lower lip length showed significant change. The mouth width increased during smiling significantly. The smile area showed no significant different in social smile between all groups. The buccal corridors area showed significant increase. The incisal show had significant difference among all groups in social smile. The smile arch depth showed no significant difference in social smile among all groups. The gingival exposure significantly increased during maximum smile. Conclusions: Control group showed moderate smile Class I with bimaxillary protrusion, spacing and openbite subgroups showed high smile. Class I with crowding and deepbite subgroups showed low smile. Class II Division 1 group showed high smile while Division 2 showed moderate smile. Class III group showed low smile.
\end{abstract}

\section{INTRODUCTION}

Smile is an important part of social interaction. It projects a variety of positive emotions such as happiness, approval and humor. As patients become more concerned with the esthetics of their smile, it has become more relevant for orthodontists to pay attention to the soft tissue framework. Recently, smile analysis has been treated as a separate
Smile analysis, lip length,

buccal corridor, smile arch, smile area.

\footnotetext{
- Paper extracted from Master's thesis entitled "Assessment of the Smile Characteristics in Different Dentoalveolar Malocclusion Cases".

1. Demonstrator, Department of Orthodontics, Faculty of Dental Medicine for Girls Al-Azhar University.

2. Professor and Head of Orthodontics Department, Faculty of Dental Medicine for Girls, Al-Azhar University.

3. Assistant Professor, Department of Orthodontics, Faculty of Dental Medicine for Girls, Al-Azhar University.
} 
entity from cephalometrics and cast analysis in orthodontic diagnosis and treatment planning. Specific hard and soft-tissue features of the smile have been studied extensively in the literature. Therefore, smile analysis is an important stage for the diagnosis, planning, treatment and prognosis of any dental treatment involving aesthetic objectives. The evaluation of the intrinsic characteristics of the smile is a necessary procedure to achieve consistent form in orthodontic treatments, which in turn makes it necessary to recognize the components and factors that affect these characteristic ${ }^{(1)}$.

Many authors ${ }^{(2-5)}$ have classified smile into different types; one author ${ }^{(2)}$ classified smile into two basic types: 1) Posed smile (social smile): it is a voluntary, unstrained, static facial expression, involving only moderate muscular contraction. The social smile is the smile typically used as a greeting $^{(3,4)}$. 2) The Duchene smile (maximum smile): it is elicited by laughter or great pleasure and it is involuntary. It results from maximal contraction of the upper and lower lip elevator and depressor muscles, respectively. This causes full expansion of the lips, with maximum anterior tooth display and gingival show ${ }^{(3,5)}$. Another author ${ }^{(1)}$ classified smile into: High Smile: where complete length of incisors is exhibited along with some amount of gingival display. Average smile: $75-100 \%$ of upper incisors and inter dental papilla are displayed. Low Smile: $<75 \%$ of the maxillary incisors in the full smile are displayed.

Smile analysis includes: a-Dentofacial analysis for Midline assessment ${ }^{(6)}$ An important finding is that most of articles agree that a small dental midline deviation does not compromise the smile esthetics and is not perceived by laypeople ${ }^{(7-9)}$. b-Dentolabial analysis: including Smile symmetry assessment ${ }^{(10)}$. An asymmetry in the smile can be due to asymmetric smile muscles' action or transverse cant of the maxillary occlusal plane ${ }^{(9)}$. The relative positioning of the corners of the mouth in the vertical plane can be assessed by the parallelism of the commissural and papillary line ${ }^{(11)}$. Lip lines assessment at rest and during function, both upper lip line and lower lip line (smile arch) are assessed. The extent of tooth display during a smile is influenced by the skeletal pattern, the mobility, and the length of the upper lip and size and positioning of the teeth ${ }^{(12-14)}$. Smile arch is the relationship between a hypothetical curve drawn along the edges of maxillary anterior teeth and inner contour of the lower lip in posed smile which is more pronounced for females than males. On the basis of this relationship, smile arches are of three types; Consonant, parallel, and reverse smile $\operatorname{arch}^{(1,3,5,15,16)}$. Buccal corridor refers to dark space (negative space) visible during smile formation between the corners of the mouth and the buccal surfaces of the maxillary teeth ${ }^{(6)}$. it could be narrow, moderate or wide buccal corridor according to arch size and the degree of muscular activity ${ }^{(5,17,18)}$. dentogingival analysis including Gingival health, contour, shape, lines(Papillary line and Cervical line $)^{(6,11,19)}$. Dental analysis including Teeth size, Axial inclination, texture, shade, Incisal embrasure, Contacts and connectors ${ }^{(6,20)}$.

The ideal is that static (photographs) image gathering should include close-up shots in frontal, sagittal and oblique planes. Smile photographs are standardized in a natural head position, with eyes looking to a distant point, during the smile photograph $^{(21)}$. Therefore, this study was directed to assess the smile characteristics in different dentoalveolar malocclusion cases, by standardized photographic analysis.

\section{SUBJECT AND METHODS}

Study sample: with the guidance of power of study, a 132 volunteer female students from Faculty of Dental Medicine for Girls, Al-Azhar University and patients from the clinic of orthodontics, Faculty of Dental Medicine for Girls, Al-Azhar University, in the time from September 2015 to June 2016.

\section{Grouping criteria}

These subjects were divided according to Angle's classification into: 1- Control group with acceptable occlusion (26 subjects) 
1- Class I ; This group was divided according to the type of malocclusion into: Bimaxillary protrusion cases (16 subjects), Crowding cases (9 subjects), Open bite cases (7 subjects), Deep bite cases (8 subjects) and Spacing cases (6 subjects)

2- Class II ; This group was divided into: Division 1 (24 subjects) and Division 2 (16 subjects) 4- Class III; (20 subjects)

\section{Methods:}

For each volunteering subject (after signing an informed consent), a spot clinical intraoral examination was done. The molar relation and the present malocclusion (If present) were denoted on each subject's sheet.

Photographic preparation: The same standardization procedure for photographic environment was set up in every session. All photos were taken by a single operator, the same chair height, the same background and fixed distance from the subject's head to the camera lens. Photographs were taken with a professional digital camera* vertically fixed over a tripod parallel to the floor. The subjects were positioned with the FH plane and the interpupillary line parallel to the floor. The imaginary center line of the patient's face was aligned to the center vertical line on the grid of the viewfinder. Via small endodontic metallic ruler, 2 marks with $2 \mathrm{~cm}$ distance had been drown on the subjects' forehead, in order to help later on image calibration. After those preparations, every subject was instructed to relax to capture the frontal rest position photo, then smile (like greeting smile) in order to capture the social smile photograph. Subsequently, a maximum smile was provoked with using a joke or social talk. Finally left profile view at rest was taken.

\section{Analysis:}

All photographs were transferred to a lap top and saved as JPEG (Joint Photographic Experts Group) image format with average size for each 5.33 MB. Afterwards, in order to complete the analysis, the images where imported to Digimizer software (Digimizer version 4.6.1 - April 14, 2016@ 20052016 MedCalc Software bvba - Ostend, Belgium). No editing procedure was done except cropping and straightening of the image, as it was noticed that few subjects tend to lean there head to the right or the left during smiling. The image was magnified by $70 \%$ of its original size in pixels. Then the calibration of measurements was done using Unit tool of the software. The 2 reference points were identified on the forehead in the image, the real length was entered $(2 \mathrm{~cm})$ and the unit was selected in the dialog box that appeared.

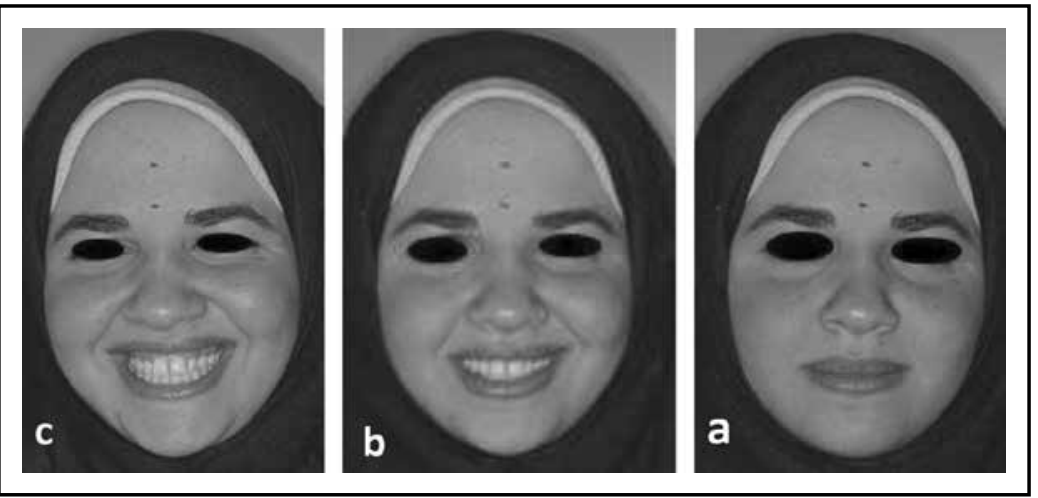

Fig. (1) Sample of standrdized extraoral photographs. a) Rest position b) Social smile c) Maximum smile

* 1Canon@1200D camera series is announced on 11 February 2014 by Canon Inc.Jaban. It is also known as the EOS Kiss X70 in Japan and the EOS Rebel T5 in the Americas. 
The following analysis items were determined:

1- Dento-facial analysis: For midline shift assessment by the guidance of image grid view option of the software.

2- Dento-labial analysis: Assessment of Upper lip length (Subnasale to Upper lip stomion ${ }^{(22,23)}$, Lower lip length from lower lip stomion to soft tissue menton ${ }^{(22)}$ and Mouth width from right to the left corner of mouth all were measured at rest and smiling (social and maximum) by linear measurement tool of the software. Smile symmetry assessment by the guidance of image grid view option. Asymmetric smile was excluded. Assessment of Smile area between the upper lip and lower lip lines by area measurement tool of the software. Assessment of Buccal corridors area which is between the posterior teeth and the

3- corner of mouth. Smile arch depth which is the vertical distance between lower lip stomion \& incisor superius ${ }^{(23)}$. Assessment of maxillary incisor display (incisal show) on both smiles by using the perpendicular line tool. It is represented by the length of line from Upper lip stomion perpendicular to incisal line.

4- Dento-gingival analysis: Gingival Height (gingival exposure) assessment by linear measuring tool from the gingival zenthis of upper right permanent central incisor and canine respectively to the upper lip line.

\section{Reliability:}

Intra-class correlations greater than or equal to 0.80 are considered to be adequately reliable. Ten percent of the images were randomly selected and re-measured by the principal investigator to test for intra-examiner reliability. All measurements yielded a Cronbach's alpha well above 0.80 . This demonstrates that the original and repeated measurements showed an acceptable level of consistency when looking at the examiner's landmark location identification. Statistical analysis: The data obtained was statistically evaluated using independent $t$-test and multiple linear regression analysis.

\section{RESULTS}

The upper lip length showed statistical significant decrease in length during both smiles. The lower lip length showed significant decrease in social smile only. The mouth width (inter-commissural distance) increased during smiling with significantly varying degrees between all groups. The smile area showed increase which was not significantly different in social smile between all groups, while it was significantly different during maximum smile. The buccal corridors area showed significant increase from social to maximum smile in all groups, while between groups the difference was not significant during social smile and significant during maximum smile. The incisal show had significant difference between all groups in social smile, with also significant change during smiling between all groups. The smile arch depth showed no significant difference in social smile between all groups, while there were significant differences during maximum smile and its change during smiling. The gingival exposure significantly increased during maximum smile and its change during smiling, while it showed no significant difference during social smile. The smile area showed weak positive correlations with the upper and lower lip length change in all groups. 
Table (1) Smile parameters for control group during rest, social smile and maximum smile.

\begin{tabular}{|c|c|c|c|}
\hline & $\begin{array}{c}\text { Rest } \\
\text { Mean (SD) }\end{array}$ & $\begin{array}{c}\text { SS } \\
\text { Mean (SD) }\end{array}$ & $\begin{array}{l}\text { Max smile } \\
\text { Mean (SD) }\end{array}$ \\
\hline Upper lip length(cm) & $2.23(0.36)$ & $1.86(0.31)$ & $1.51(0.26)$ \\
\hline Lower lip length(cm) & $4.55(0.59)$ & $4.21(0.66)$ & $4.31(0.50)$ \\
\hline Mouth width $(\mathbf{c m})$ & $5.54(0.47)$ & $6.34(0.46)$ & $6.91(0.64)$ \\
\hline Smile area $\left.\mathbf{c m}^{2}\right)$ & & $3.58(1.23)$ & $6.04(1.52)$ \\
\hline Buccal corridors area $\left.\mathbf{c m}^{\mathbf{2}}\right)$ & & $0.14(0.09)$ & $0.37(0.14)$ \\
\hline Buccal corridors to smile area ratio & & $0.04(0.02)$ & $0.06(0.05)$ \\
\hline Incisal show $(\mathbf{c m})$ & & $0.75(0.21)$ & $1.06(0.26)$ \\
\hline Smile arch depth(cm) & & $0.11(0.08)$ & $0.25(0.14)$ \\
\hline Gingival exposure at upper 1(cm) & & $0.06(0.03)$ & $0.24(0.16)$ \\
\hline Gingival exposure at upper 3(cm) & & $0.03(0.08)$ & $0.21(0.17)$ \\
\hline
\end{tabular}

Table (2) Smile parameters in Malocclusion groups during rest, social smile and maximum smile.

\begin{tabular}{|c|c|c|c|c|}
\hline & & Rest Mean (SD) & SS Mean (SD) & $\operatorname{Max} \mathbf{S}$ Mean (SD) \\
\hline \multirow{8}{*}{ Upper lip length(cm) } & Bimax.pro & $2.67(0.46)$ & $2.06(0.50)$ & $1.63(0.45)$ \\
\hline & Crowding & $2.28(0.27)$ & $1.88(0.29)$ & $1.99(0.18)$ \\
\hline & Deep bite & $2.49(0.53)$ & $2.06(0.53)$ & $1.60(0.45$ \\
\hline & Open bite & $2.61(0.32)$ & $1.86(0.15$ & $1.84(0.31)$ \\
\hline & Spacing & $2.23(0.27)$ & $1.85(0.26$ & $1.87(0.37)$ \\
\hline & Class II div1 & $2.45(0.37$ & $1.97(0.43$ & $1.66(0.27)$ \\
\hline & Class II div2 & $2.28(0.15)$ & $1.82(0.24)$ & $1.61(0.19)$ \\
\hline & Class III & $2.27(0.35)$ & $1.82(0.35)$ & $1.51(0.29)$ \\
\hline \multirow{8}{*}{ Lower lip length $(\mathrm{cm})$} & Bimax.pro & $4.76(0.43)$ & $4.72(0.27)$ & $4.53(0.32)$ \\
\hline & Crowding & $4.62(0.32)$ & $4.31(1.11)$ & $4.31(1.19)$ \\
\hline & Deep bite & $4.99(0.59)$ & $4.74(0.52)$ & $4.64(0.48)$ \\
\hline & Open bite & $4.95(0.25)$ & $4.34(0.16)$ & $4.45(0.34)$ \\
\hline & Spacing & $5.01(0.57)$ & $4.74(0.56)$ & $5.02(1.11)$ \\
\hline & Class II div1 & $4.44(0.57)$ & $4.09(0.86)$ & $4.12(0.72)$ \\
\hline & Class II div2 & $4.28(0.56)$ & $4.19(0.57)$ & $4.37(0.56)$ \\
\hline & Class III & $4.86(0.66)$ & $4.93(0.85)$ & $4.68(0.95)$ \\
\hline \multirow{8}{*}{ Smile $\operatorname{area}\left(\mathrm{cm}^{2}\right)$} & Bimax.pro & & $5.74(1.73)$ & $8.46(1.51)$ \\
\hline & Crowding & & $3.35(0.64$ & $6.46(1.8$ \\
\hline & Deep bite & & $3.91(0.86)$ & $6.53(1.75)$ \\
\hline & Open bite & & $4.98(2.38$ & $7.49(2.34)$ \\
\hline & Spacing & & $5.58(3.06)$ & $8.40(2.2$ \\
\hline & Class II div1 & & $4.33(2.03$ & $6.96(2.31)$ \\
\hline & Class II div2 & & $3.45(1.14)$ & $5.96(2.29)$ \\
\hline & Class III & & $3.73(0.99)$ & $6.77(2.23)$ \\
\hline
\end{tabular}




\begin{tabular}{|c|c|c|c|c|}
\hline & & Rest Mean (SD) & SS Mean (SD) & Max S Mean (SD) \\
\hline \multirow{8}{*}{$\begin{array}{l}\text { Buccal } \\
\text { corridors } \\
\text { area }\left(\mathrm{cm}^{2}\right)\end{array}$} & Bimax.pro & & $0.17(0.09)$ & $0.42(0.12)$ \\
\hline & Crowding & & $0.14(0.09)$ & $0.33(0.22)$ \\
\hline & Deep bite & & $0.27(0.19)$ & $0.64(0.33)$ \\
\hline & Open bite & & $0.15(0.02)$ & $0.38(0.11)$ \\
\hline & Spacing & & $0.09(0.02)$ & $0.35(0.14)$ \\
\hline & Class II div1 & & $0.35(0.53$ & $0.56(0.14)$ \\
\hline & Class II div2 & & $3.45(1.14)$ & $5.96(2.29)$ \\
\hline & Class III & & $0.12(0.04)$ & $0.44(0.14)$ \\
\hline \multirow{8}{*}{ Buccal corridors area } & Bimax.pro & & $0.02(0.02)$ & $0.05(0.04)$ \\
\hline & Crowding & & $0.09(0.07)$ & $0.05(0.03)$ \\
\hline & Deep bite & & $0.07(0.05)$ & $0.07(0.05)$ \\
\hline & Open bite & & $0.03(0.01)$ & $0.05(0.02$ \\
\hline & Spacing & & $0.02(0.02)$ & $0.05(0.03)$ \\
\hline & Class II div1 & & $0.09(0.08)$ & $0.11(0.73)$ \\
\hline & Class II div2 & & $0.10(0.06)$ & $0.29(0.16)$ \\
\hline & Class III & & $0.03(0.02)$ & $0.05(0.04)$ \\
\hline
\end{tabular}

\section{DISCUSSION}

Evaluating beauty is always subjective. Therefore, the establishment of norms is an important part in orthodontic diagnosis and treatment planning. It would be prudent to evaluate the parameters of smile before treatment in order to know not only what needs to be done, but also what can be done, and to communicate that with patients and/or parents. In this study, Intra and intergroups comparisons and correlations were done in order to explore the influence of the different types of dentoalveolar malocclusions on the smile characteristics in addition to providing a theoretical basis for clinical diagnosis and treatment planning.

In the current study, In control group the mean upper lip length was $(2.23 \mathrm{~cm} \pm 0.36)$ at rest position. This was in accordance with the reported average $(2.2 \mathrm{~cm} \pm 0.2)^{(25)}$. During smiling, the highest upper lip length was in Class I(bimaxillary protrusion) this was in contrast to other study which reported that the upper lip length in Class II Div1 was higher compared to Class I and Class II Div2 ${ }^{(26)}$. while at maximum smile the least mean value was recorded in Class III. These results came in accordance with other previous studies ${ }^{(27)}$. This current study results was consistent with the idea that protrusion of upper incisors in Class I (bimaxillary protrusion) and Class II cases may cause decreasing of the lip elasticity and the muscle ability to raise the upper lip ${ }^{(26)}$.

Regarding the lower lip length, in this study, in control group at rest was $(4.55 \mathrm{~cm} \pm 0.5)$, while during both, social and maximum smiles were $(4.2 \mathrm{~cm} \pm 0.6$, $4.31 \mathrm{~cm} \pm 0.5)$ respectively. These results were not consonant with a former studies which reported that the lower lip length in maximum smile is $3.77 \mathrm{~cm}$, however these studies didn't discriminate between different types of malocclusion ${ }^{(28,29)}$. In this study, the highest values of lower lip length during social and maximum smiles were recorded in Class III and Class I (spacing) subjects respectively. While the least values at rest and smiling were recorded in Class.

These results were in accordance with other studies which showed that, Class III subjects possess highest value of lower lip length followed by Class II Div1, Class II Div2 subjects and Class I subjects respectively ${ }^{(27)}$. This may be due to lack of interdigitation which 
opens vertical height. Decreased lower one third height is associated with vertical maxillary deficiency and mandibular retrusion deep bites.

The current study revealed that in control group, the mean values of percent change in mouth width from rest position to social and maximum smile respectively were $(14.7 \% \pm 7.5,24.9 \% \pm 8.7)$. A former study showed that the mean percent change of mouth width in maximum smile was $30 \%{ }^{(29)}$. The highest mean value of mouth width during both smiles were recorded in Class I (bimaxillary protrusion) while the least values were in Class I (crowding).

Regarding smile area, In control group, the mean values of social and maximum smile area were $\left(3.5 \mathrm{~cm}^{2} \pm 1.2,6.04 \mathrm{~cm}^{2} \pm 1.5\right)$ respectively. The highest mean values of smile area were recorded in both smiles in Class I (Bimaxillary protrusion) while the least smile area mean values in social smile were in Class I (crowding) and Class II Div 2 in maximum smile. These findings could be explained as results of the fact that the soft tissue structure and behavior follow the dentoalveolar base. By searching literatures- According to this study searching protocol- there was nearly no previous study that compare the smile area and its percent change in different dentoalveolar malocclusion cases. But there was a study that correlated the smile area to the sum of right and left buccal corridors ${ }^{(30)}$.

Regarding the sum of the right and left buccal corridors area, The highest buccal corridors area during social and maximum smile were recorded in Class I (crowding and deep bit respectively), while the lowest values were recorded during both smiles on Class II Div 2. This results was in contradiction with a study that had stated that Buccal corridor space is larger in individuals with Class II Division 1 malocclusion when compared with individuals with Class I malocclusions ${ }^{(4)}$. By searching literatures-

According to this study searching protocol- there was nearly no previous study that evaluated the buccal corridor as an area in different dentoalveolar malocclusion cases.
In this study, the ratio between the sum of right and left buccal corridors area to the smile area in control group were in social and maximum smiles $(0.04 \pm 0.02,0.06 \pm 0.05)$ respectively. The highest mean values were recorded during social smile in Class I (crowding) followed by Class II Div 1 and the lowest was in Class I (spacing). While in maximum smile it was the highest in Class II Div 1 and lowest in Class II Div 1. Those results were in accordance with a previous study ${ }^{(31)}$.

In the current study, The highest incisal show was present during both, social and maximum smile in Class I (bimaxillary protrusion). The lowest incisal show was in Class I (crowding) in social smile and Class III in maximum smile, while the highest percent change was recorded in Class II Div 1. Other previous study found a decrease in incisor display at rest and smiling in proclined maxillary incisors, whether in a Class II Div 1 malocclusion or in a Class III compensation ${ }^{(18)}$.

The optimal smile arch is described as "consonant", the curvature of the maxillary incisal edges coincides with or parallels the border of the lower lip in smiling. ${ }^{(12,18)}$ In this study, the mean values of smile arch depth in control group were $(0.11 \mathrm{~cm} \pm 0.08$ and $0.25 \mathrm{~cm} \pm 0.14)$ in both social and maximum smile respectively, with $281.4 \%$ mean percent change from social to maximum smile. In the current study, the deepest smile arch values were recorded in Class II Div1 in social smile and Class I (open bite) in the maximum smile and the shallowest (parallel) was in class I deep bite. In Two previous studies ${ }^{(15)}$,both found the parallel smile arch to be most frequent in their subjects.

Regarding the gingival exposure, it has been measured in two regions, at the upper right central incisor and the upper right canine. In the upper central region, the highest gingival exposure was recorded in Class I (bimaxillary protrusion) during both social and maximum smile. However, in the canine region the highest values were recorded during social smile in Class II Div2, and Class I (bimaxillary protrusion) during maximum smile. 


\section{CONCLUSIONS}

Within the limits of this study,Control group was characterized by average low upper lip length at rest. During both smiles there were low smile area, consonant smile arch, moderate to low buccal corridors area and average incisal and gingival exposure.

\section{Class I with Bimaxillary protrusion subgroup} was characterized by Long upper lip at rest with all criteria of the high smile.

Class I with Crowding subgroup was characterized by Low mouth width and incisal show during social smile, low smile area in both smiles, high buccal corridor to social smile area ratio. Class I with Deepbite subgroup was characterized by increased buccal corridor area, low buccal corridor to smile area ratio and consonant smile arch all were during maximum smile. Class I with Openbite subgroup was characterized by; The highest mouth width at rest, revers smile arch and high gingival exposure at upper central incisor both were during maximum Class I with Spacing subgroup was characterized by: Increased smile area during maximum smile. During social smile, there were low buccal corridor to smile area ratio and low gingival exposure at upper central incisor and canine. Class II Division 2 group was characterized by; The shortest lower lip length during social smile, high buccal corridor area and its ratio to smile area during both smiles. Class II Division 2 group was characterized by: The lowest mouth width at rest, decreased buccal corridor area and its ratio to smile area both during social smile. Class III group was characterized by: the least upper lip length at rest, the highest lower lip length and decreased buccal corridor area both were during social smile, with decreased incisal show during maximum smile.

\section{REFERENCES}

1. McNamara L, McNamara JA Jr, Ackerman MB, Baccetti T. Hard and soft-tissue contributions to the esthetics of the posed smile in growing patients seeking orthodontic treatment. Am J Orthod Dentofacial Orthop 2008; 133:491-9.
2. Ackerman M.B, Brensinger C., Landis J.R. An evaluation of dynamic lip-tooth characteristics during speech and smile in Adolescents. Angle Orthod.2004;74:43-50.

3. Ackerman MB, Ackerman JL. Smile analysis and design in the digital era. J Clin Orthod. 2002; 36:221-36.

4. Bhat R, Ravi M. Factors affecting buccal corridor space in Angle's Class II Division 1 malocclusion. J Orof Sci 2014; 31:56-67.

5. Sarver D.M. The importance of incisor positioning in esthetic smile: The smile Arc. Am J Orthod Dentofacial Orthop 2001; 120:98-111.

6. Singla S, Gurvanit L S, Lehl G. Smile analysis in orthodontics (a review article). Indian J Oral Sci. 2014; 5:49-54.

7. Rodrigues CD, Magnani R, Machado MS, Oliveira OB. The perception of smile attractiveness. Angle Orthod. 2009; 79: 634-39.

8. Pinho S, Ciriaco C, Faber J, Lenza M. Impact of dental asymmetries on the perception of smile esthetics. Am J Orthod Dentofacial Orthop. 2007; 132:748-53.

9. Ker AJ, Chan R, Fields HW, Beck M, Rosenstiel S. Esthetics and smile characteristics from the layperson's perspective: a computer-based survey study. J Am Dent Assoc 2008; 139:1318- 27.

10. Ritter DE, Gandini LG, Pinto Ados S, Locks A. Esthetic influence of negative space in the buccal corridor during smiling. Angle Orthod. 2006; 76:198-203.

11. Roy S.The eight components of a balanced smile, J Clin Orthod. 2005; 39:155-67.

12. Garber DA, Salama MA., The aesthetic smile: Diagnosis and treatment. Periodo J. 2000; 11:18- 28.

13. Teo CS. An evaluation of the smiling lip line. Singapore Dent J. 1981; 6:27-30.

14. Goldstein RE. Digital dental photography now. Contemp Esthet Restor Pract. 2005;9:12-5.

15. Tjan AH, Miller GD, The JG. Some esthetic factors in a smile. J Prosthet Dent. 1984; 51:24-8.

16. Câmara C. A. Aesthetics in Orthodontics: Six horizontal smile lines. Dental Press J Orthod. 2010; 119:118-31.

17. Machado AW. 10 commandments of smile esthetics. Dental Press J Orthod. 2014; 19:136-57.

18. Sarver DM, Ackerman MB. Dynamic smile visualization and quantification: Part 2. Smile analysis and treatment strategies. Am J Orthod Dentofacial Orthop. 2003; 124:116-27. 
19. Sharma PK, Sharma P. Dental smile esthetics: The assessment and creation of the ideal smile. Semin Orthod. 2012; 18:193-201.

20. Priya K. Rahul D P, Varma S, Namitha R. Norms for Crafting a Beautiful Smile. Amrita J Med. 2013; 9:1-44.

21. Kiran DN, Anupama DK. Digital photography in dentistry. Indian J Stomatol. 2010; 1:77-80.

22. Edler RJ. Background Considerations to Facial Aesthetics. J Orthod. 2001;23:159-68.

23. Perkins RA, Staley RN. Change in lip vermilion height during orthodontic treatment. Am J Orthod Dentofacial Orthop 1993; 103:147-54.

24. Robert TB. Cephalometric soft tissue facial analysis. Am J Orthod Dentofacial Ort-hop.1999; 116: 373-89.

25. Rifkin R: Facial analysis: A comprehensive approach to treatment planning in aesthetic dentistry. Pract Periodontics Aesthet Dent 2000; 12:865-72.

26. Islam R, Kitahara T, Naher L, Hara A, Nakasima A Lip morphological changes in orthodontic treatment. Class II division 1: malocclusion and normal occlusion at rest and on smiling. Angle Orthod. 2009; 79: 256-64.

27. Al-Hamdany AKH. Integumental lips' height and separation in different Angle's classes of malocclusions. Al-Rafidain Dent J. 2007; 7:38-49.

28. Rakosi Th. Rakosi T. An atlas and manual of cephalometric radiography. $1^{\text {st }}$ ed. Landone. Wolf Medical Publications Ltd. 1982.

29. Ra'uf FMS. Facial analysis and facial types of the students in Mosul University, normal occlusion. The direct method. MSc Thesis. College of Dentistry. University of Mosul. 1997.

30. kakadiya J., Pattnaik B , Kumari M., Vishnoi P. An Evaluation of smile in different malocclusion of local population, A pilot study. J Dent Med Sci. 2015; 14:25-32.

31. Zhang L, Lu H Y. Study on the Smile Characteristics of Patients with Different Types of Malocclusion. Master's thesis, Hebei Medical University; 2014. 